\title{
大展弦比飞机几何非线性气动弹性稳定性的 线性化方法
}

\author{
谢长川 ${ }^{*}$ ，杨超 \\ 北京航空航天大学航空科学与工程学院, 北京 100191 \\ *E-mail: xiechangc@163.com
}

收稿日期: 2010-09-20; 接受日期: 2010-11-23

国家自然科学基金(批准号: 90716006, 10902006)和高等学校博士点基金(批准号: 20091102110015)资助项目

\begin{abstract}
摘要 基于动力学小扰动假设建立了具有大展弦比机翼柔性飞机的全机几何非线性气动弹 性稳定性分析的线性化方法和工程求解流程, 并通过复杂算例验证了该方法的工程适用性. 对 某高空长航时无人机, 计算了飞机在平飞设计载荷以及阵风载荷作用下的非线性静变形, 在对 应的非线性平衡态下对全机进行动力学线性化, 计算了考虑静态大变形因素的全机固有振动 特性, 采用偶极子格网法计算了非定常气动力, 进一步分析了全机的气动弹性稳定性, 并与传 统线性计算结果进行了对比研究. 计算结果表明, 由于结构大变形引起的几何非线性会引起机 翼面内弯曲和扭转的运动耦合, 改变相应模态的频率和振型, 从而影响气动弹性耦合关系, 降 低颤振临界速度. 传统的线性方法不但不能得到准确的颤振临界速度, 而且有可能给出错误的 稳定性结论. 因此, 对于具有大展弦比机翼的高空长航时无人机, 以及类似的大柔性飞行器, 必须在其设计过程中进行几何非线性气动弹性稳定性分析.
\end{abstract}

关键词 气动弹性 几何非线性 颤振 大变形

\section{1 引言}

近十几年来, 高空长航时飞机引起了广泛的研 究兴趣, 具有大展弦比机翼的全机气动弹性稳定性 成为航空工程师尤为关注的重要课题. 在 20 世纪 90 年代早期, van Schoor 等人 ${ }^{[1]}$ 基于完全线性理论对一 个大柔性飞机进行了气动弹性分析, 讨论了结构弹 性对气动弹性和飞行动力学稳定性的影响. Patil 和 Hodges $^{[2,3]}$ 首先将几何非线性引入到飞机气动弹性问 题当中, 他们采用非线性有限元方法对系统进行了 稳定性分析并计算了系统极限环, 他们所用的完全 非线性方法适用于较为简单模型的响应分析.
Dowell 等人 ${ }^{[4]}$ 清楚的指出, 对于同时具有结构 和气动非线性的特别大展弦比机翼是重要的研究课 题, 同时指出 “静态非线性而动态线性, $\cdots \cdots$, 那么 流体-结构 (气动弹性)系统动力稳定性问题就能够通 过在非线性平衡态附近进行线性动态扰动分析来实 现”. 对复杂结构, 有限元方法给出的自由度数过多, 这使得系统过稳定运动, 如极限环的计算非常困难 而且效率很低. 因此, 动力学线化方法就成为确定机 翼或全机气动弹性临界稳定条件的最为有效的工程 分析手段.

在过去的十几年中, 以梁式机翼作为大展弦比 机翼最为简化的模型被广泛的用来研究其气动弹性 
行为的机理. 针对梁式模型, Patil 和 Hodges ${ }^{[5]}$ 采用动 力学线性化方法研究了几何非线性效应对静、动气动 弹性行为的影响, 该模型的机翼面内弯曲频率高于 机翼扭转频率, 几何非线性引起了这两阶模态的运 动耦合, 使得扭转频率随载荷幅值增大而降低, 从而 相应的颤振速度也会减小, 研究表明非定常气动力 的非平面效应即使在较大的机翼变形量下也是可以 忽略的因素. Tang 和 Dowell ${ }^{[6]}$ 针对梁式机翼模型进 行了风洞试验研究, 对比了理论计算和试验结果, 研 究表明线性化方法能够精确的预测颤振临界速度. 谢长川 ${ }^{[7]}$ 采用推广的片条理论对金属单梁式机翼进 行了非线性静气动弹性变形计算, 并采用线性化方 法预测了颤振临界速度, 计算与风洞试验结果具有 很好的一致性. 张健 ${ }^{[8]}$ 采用完全非线性梁模型和气动 力的 ONERO 方法对梁式模型进行了较为深入的研究, 得到了颤振临界速度和失稳后的极限环运动. 在单独 机翼研究的基础之上, Hodges 及其学生 Patil 和 Cesnik 的研究团队进一步采用组合梁模型对全机几何非线 性气动弹性和飞行动力学稳定性问题进行了广泛的 研究 $^{[3,9 \sim 11]}$. 然而, 当前研究普遍采用的简化模型和 完全非线性算法对于工程实际应用还难以实现.

近来, 几何非线性气动弹性分析的动力学线化 方法逐渐被应用于工程分析领域. Strong 等人 ${ }^{[12]}$ 粗略 的研究了预应力作用下的复杂机翼, 并与完全线性 的计算结果进行了对比. 谢长川等人 ${ }^{[13 \sim 15]}$ 也采用线 性化方法分别研究了大展弦比金属双梁机翼和复合 材料机翼的颤振特性, 机翼大变形的几何非线性使 得面内弯曲与扭转产生运动耦合, 随着载荷和变形 量的增加, 这两阶模态中频率较低的进一步下降而 频率较高的则会增加, 同时面内弯曲振型具有明显 的扭转分量而扭转振型具有面内弯曲分量. 对于各 向同性的金属材料机翼, 通常其面内弯曲频率高于 扭转频率, 考虑几何非线性后扭转频率下降从而使 得弯扭耦合颤振速度比线性情况降低; 而对于复合 材料机翼, 其面内弯曲刚度则是传统设计中不受重 视的因素, 有出现面内弯曲频率远低于扭转频率的 可能, 在考虑几何非线性后具有较大扭转分量的面 内弯曲模态会引起相当严重的非线性气动弹性问题.

本文研究一个具有插入式大展弦比复合材料机 翼的高空长航时无人机, 其机翼具有较大变形量. 首 先对其进行非线性静力学平衡态计算, 然后在该平 衡态对系统进行动力学线性化, 从而确定全机的几
何非线性气动弹性稳定性.

\section{2 结构和气动弹性理论}

对高空长航时无人机的大展弦比机翼, 在爬升 机动甚至在定直平飞过程中都会产生相当大的变形, 必须在指定飞行状态下考虑结构变形后的构型上进 行结构静力学和动力学分析, 此时飞行载荷和几何 非线性具有相当重要的作用. 因此, 飞行器的气动弹 性稳定性与线性情况相比可能出现较大的改变. 求 解完全的非线性气动弹性问题通常相当困难, 然而 对于亚音速气动弹性稳定性而言很多因素都能够忽 略. 可以假定：1）结构在其非线性静平衡状态附近 做微幅振动；2）由于大攻角引起的气动非线性可以 忽略, 因为这受到飞行载荷和失速要求的设计限制; 3) 非定常气动力的非平面效应可以忽略不计. 在这 些假设条件之下, 气动弹性稳定性分析中传统的频域 方法经过适当的修改就可以得以应用. 在计算得到结 构非线性静力学平衡态后, 可以求得结构的固有振动 模态, 由此能够采用偶极子格网法计算频域气动力, 然后通过 $p-k^{\text {法 }}{ }^{[16]}$ 给出系统的失稳临界速度.

\section{1 几何非线性弹性力学}

弹性细长机翼承受较大气动力时产生有限弯曲 和扭转变形, 因此小变形假设不再满足, 但是结构材 料仍然认为没有超出小应变范围, 这会产生几何非 线性弹性力学方程, 其中包括了位移微分的二次项, 并且需要在结构变形后的构型下建立非线性的力平 衡方程. 结构几何非线性问题通常可以采用增量有 限元方法进行求解, 包括完全 Lagrange 格式(TLF)和 更新 Lagrange 格式(ULF) ${ }^{[17]}$. 本文工作中采用 ULF 方法.

Lagrange/Green 应变与位移的非线性关系为

$$
{ }^{t} \varepsilon_{i j}=\frac{1}{2}\left({ }^{t} u_{i, j}+{ }^{t} u_{j, i}+{ }^{t} u_{k, i}{ }^{t} u_{k, j}\right),
$$

其中, ${ }^{t} u_{i, j}$ 为时刻 $t$ 位移 $u_{i}$ 对坐标 $x_{j}$ 的偏导数. 对偶 的 $t$ 时刻 Kirchhoff 应力张量满足

$$
{ }^{t} S_{j i}{ }^{t} n_{j}{ }^{t} \mathrm{~d} s={ }^{t} x_{i, j}{ }^{t} \mathrm{~d} T_{j},
$$

其中, ${ }^{t} n_{j}$ 为微小面元 $\mathrm{d} s$ 在时刻 $t$ 的方向余弦; $\mathrm{d} T_{j}$ 为对 应的表面力, 这里考虑了随动力效应. 线性的弹性本 构关系仍可表示为 


$$
{ }^{t} S_{i j}={ }^{t} D_{i j k l}{ }^{t} \varepsilon_{k l},
$$

$D_{i j k l}$ 是弹性张量, 对各向同性材料和各向异性材料具 有不同的形式.

\section{2 有限元法}

基于能量原理的有限元法(FEM) 是求解结构问 题的有效工具, 对于具有随动载荷效应的几何非线 性问题可以采用增量有限元方法. 将应变 $\varepsilon_{i j}$ 分解为 线性部分 $e_{i j}$ 和非线性部分 $\eta_{i j}$,

$$
{ }^{t} \varepsilon_{i j}={ }^{t} e_{i j}+{ }^{t} \eta_{i j} .
$$

应力则按增量进行分解, 其中 ${ }^{t} S_{i j}$ 表示 $t$ 时刻的 平衡应力, ${ }^{t} \tau_{i j}$ 表示同一时刻的应力增量.

$$
{ }^{t+\Delta t} S_{i j}={ }^{t} S_{i j}+{ }^{t} \tau_{i j} .
$$

在每一增量步进行线性化可得如下积分方程,

$$
\begin{aligned}
& \int_{{ }^{t} V}{ }^{t} D_{i j k l}{ }^{t} e_{k l} \delta^{t} e_{i j} \mathrm{~d}^{t} V+\int_{{ }^{t} V}{ }^{t} \tau_{i j} \delta^{t} \eta_{i j} \mathrm{~d}^{t} V \\
& ={ }^{t+\Delta t} Q-\int_{{ }^{t} V}{ }^{t} \tau_{i j} \delta^{t} e_{i j} \mathrm{~d}^{t} V,
\end{aligned}
$$

其中, ${ }^{t+\Delta t} Q$ 为新增量步时刻的外力增量, 可以包括 气动力、发动机推力和重力等. 引入一系列型函数, 位移与应变关系可以表示为

$$
{ }^{t} e={ }^{t} B_{L}{ }^{t} u, \quad{ }^{t} \eta={ }^{t} B_{N L}{ }^{t} u .
$$

将其带入方程(6)可得单元控制方程形如

$$
\left({ }^{t} K_{L}+{ }^{t} K_{N L}\right) u={ }^{t+\Delta t} \bar{Q}-{ }^{t} F,
$$

相应的动力学方程为

$$
{ }^{t} M^{t+\Delta t} \ddot{u}+\left({ }^{t} K_{L}+{ }^{t} K_{N L}\right) u={ }^{t+\Delta t} \bar{Q}-{ }^{t} F .
$$

\section{3 动力学线性化}

在许多动力学问题中仍然可以认为结构在其静 平衡态附近做微幅振动, 这其中就包括了动力学稳 定性问题. 可以记为

$$
u=\bar{u}+x,
$$

其中, $\bar{u}$ 是由方程(8)计算得到的静态平衡大变形; $x$ 为微幅振动位移. 依据方程(9)和静平衡条件, 系统 在定常载荷作用下的自由振动方程可以表示为

$$
\boldsymbol{M}_{T} \ddot{x}+\boldsymbol{K}_{T} x=0,
$$

其中, $\boldsymbol{M}_{T}$ 是结构静平衡构型下的惯性矩阵; $\boldsymbol{K}_{T}$ 是相应 的刚度矩阵. 由方程(11)即可得到各阶振型和频率.

\section{4 非定常气动力}

非定常气动力计算采用亚音速偶极子格网法, 这里忽略了变形后气动面的非平面效应，并且局部攻 角小于其失速极限. 每一气动单元的非定常压强为

$$
\Delta p=\frac{1}{2} \rho V^{2} C^{-1}\left(\phi_{H}^{\prime}+\mathrm{i} \frac{k}{b} \phi_{H}\right) q,
$$

其中, $\rho$ 为指定高度的大气密度; $V$ 为来流速度; $\phi_{H}$ 为 气动单元控制点处的模态振型法向分量; $\phi_{H}^{\prime}$ 为气动 单元控制点处模态振型沿来流方向的斜率; $q$ 为结构 模态的广义坐标; $k=b \omega / V$ 是减缩频率, $\omega$ 是圆频率, $b$ 是参考弦长; $C$ 是气动力影响系数矩阵. 当减缩频 率为零时即得到定常气动力. 气动力计算的详细理 论可参考文献[16, 18].

\section{5 配平分析}

弹性飞机的飞行载荷配平计算通过全机静气动 弹性方程来求解, 经过有限元离散化写为

$$
\boldsymbol{K} \bar{u}+\boldsymbol{M} a=\frac{1}{2} \rho V^{2} \boldsymbol{Q}_{u} \bar{u}+\frac{1}{2} \rho V^{2} \boldsymbol{Q}_{\delta} \delta+P_{0},
$$

这里, $\boldsymbol{K}$ 为结构刚度矩阵, 在考虑结构几何非线性时 它是结构位移和载荷状态的非线性函数; $\boldsymbol{M}$ 仍为结构 惯性矩阵; $a$ 为结构加速度, 它仅代表了飞机刚体自 由度的加速度; $\boldsymbol{Q}_{u}$ 为结构弹性变形引起的气动力影 响系数矩阵; $\boldsymbol{Q}_{\delta}$ 为机体姿态和舵面偏转引起的气动 力影响系数矩阵; $P_{0}$ 为其他外部载荷, 例如重力等.

考虑飞机结构具有较大变形时飞机静气动弹性 问题是复杂的非线性方程, 直接求解具有很多困难 之处. 在实际处理过程中往往需要进行较大的简化, 例如忽略气动面的非平面效应等，然后再构造适当 的迭代流程得到方程(13)的近似解.

\section{6 颤振分析}

选取一系列结构模态，可以建立频域的广义气 动弹性方程,

$$
\boldsymbol{M} \ddot{q}+\boldsymbol{K} q=\frac{1}{2} \rho V^{2} \boldsymbol{A} q,
$$

其中, $\boldsymbol{M}$ 为广义质量矩阵; $\boldsymbol{K}$ 为广义刚度矩阵; $\boldsymbol{A}$ 为广 义气动力影响系数矩阵, 它是减缩频率的复值函数.

像传统颤振分析中一样方程(14)仍可采用 $p-k$ 法进行求解. 可将方程变换为如下广义特征值问题 的形式, 


$$
\left[p^{2} \boldsymbol{M}-p \frac{b}{2 k} \rho V \boldsymbol{A}^{I}+\left(\boldsymbol{K}-\frac{1}{2} \rho V^{2} \boldsymbol{A}^{R}\right)\right] q=0,
$$

其中, $\boldsymbol{A}^{R}$ 和 $\boldsymbol{A}^{I}$ 分别为 $\boldsymbol{A}$ 的实部和虚部; $p=\omega(\gamma \pm i)$ 是 系统在给定速度 $V$ 时的复特征值. 结构阻尼系数 $g=2 \gamma$, 频率则为 $f=\omega / 2 \pi$. 通过 $V-g$ 曲线可以确定 系统的颤振临界速度(在该速度点 $g$ 值由负变正), 相 应在 $V-f$ 曲线上能够得到颤振频率 ${ }^{[16]}$.

\section{3 分析流程}

由于考虑结构几何非线性的系统动力学特性受 其变形和应力状态影响, 因此具有大展弦比机翼的 全机气动弹性稳定性问题应综合其静力学和动力学 进行一体化求解. 由此, 真实系统的有限元模型应该 能够给出真实的结构变形和应力状态, 同时给出其 动力学特性.

可以建立如图 1 所示的稳定性分析流程. 首先, 在给定的飞行条件下迭代求解全机非线性静力学, 得到静气动弹性分布载荷以及配平状态. 其次, 在上 一步分析所得的静力学平衡态上计算其振动模态和 频率, 以及相应的非定常气动力. 然后就可以采用传 统的颤振分析方法判断系统在该状态下的稳定性. 最后, 对飞行速度进行迭代计算并重复前两步骤, 从 而确定系统失稳边界. 但是在很多情况下不同的飞

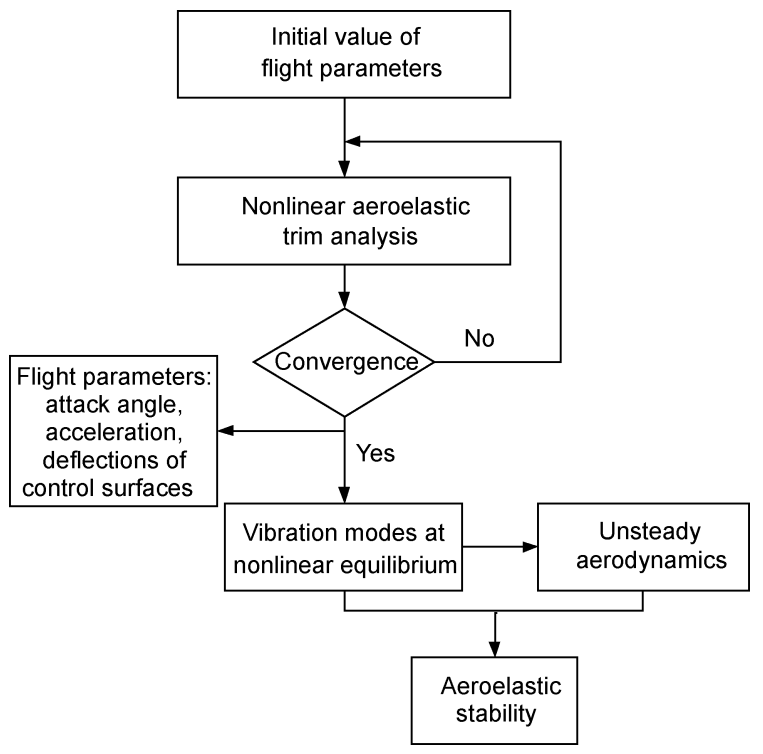

图 1 非线性气动弹性稳定性分析流程图
行条件也会改变系统的气动弹性特性, 例如过载系 数, 机动类型等.

在本文中, 研究目标并不是要给出详细的全机 非线性载荷分布以及非线性配平分析，而是要确定 全机在预载荷作用而具有有限变形情况下的动力学 特性和动气动弹性稳定性. 因此, 文中仅以全机设计 载下的线性配平情况作为初始状态，非线性配平分 析时仅以全机加速度作为配平参数. 然后在非线性 平衡态下计算全机振动模态振型和频率, 从而分析 全机考虑几何非线性情况颤振耦合机理.

\section{4 算例研究}

本文选取一个具有大展弦比复合材料机翼的飞 机来研究说明结构几何非线性对全机气动弹性稳定 性的影响. 全机结构有限元模型如图 2 所示, 机翼全 展长约为 $19.0 \mathrm{~m}$, 展弦比约为 18.0 , 平均后掠角约为 $5^{\circ}$, 全机单元数约为 30000 . 机翼材料铺层设计为使 机翼产生外洗, 即向上弯曲变形同时引起抬头扭转 [19]. 机翼部件的非线性气动弹性稳定性分析参见文 献[14]. 全机气动力模型如图 3 所示, 这里忽略了机 身气动力.

全机分析分为线性情况和非线性情况以便于对 比. 全机线性气动弹性稳定性与其变形和载荷状态

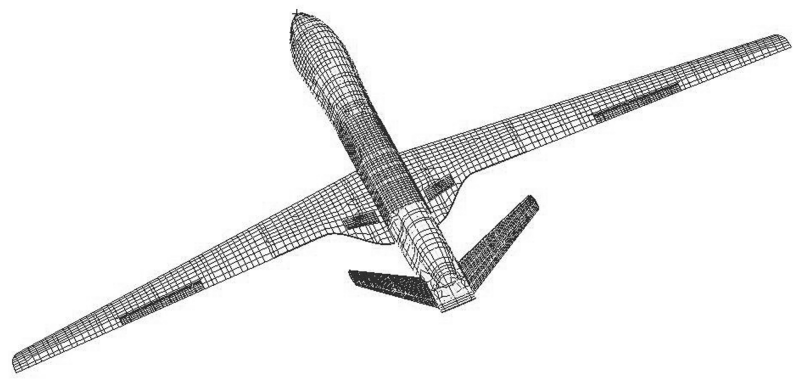

图 2 全机有限元模型

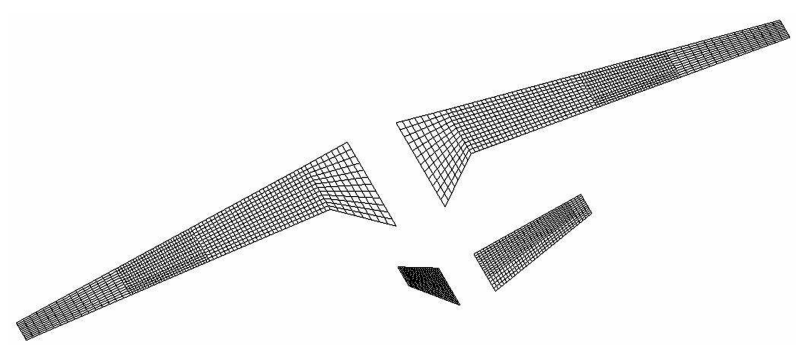

图 3 全机气动力模型 
无关; 而非线性分析中选用一个标称飞行状态, 本文 中称该状态为设计载荷情况. 设计载荷的飞行动压 记为 $q_{\mathrm{D}}$, 对应的海平面飞行速度为 $V_{\mathrm{D}}$. 在静力学分 析中定常气动力始终垂直于气动面, 即考虑了所谓 的“随动载荷”效应.

\section{1 线性情况}

采用线性气动弹性方法, 对全机进行了平飞速 度为 $V_{\mathrm{D}}$ 的配平计算, 表 1 给出了平飞设计载荷情况 下的翼尖变形量, 其中相对变形为变形量除以半弦 长. 可以看到飞机平飞时翼尖垂直位移大于半展长 的 $5 \%$, 具有较大的弯曲变形, 但翼尖弹性攻角小于 $2^{\circ}$, 变形后飞机仍能维持其气动性能.

依据线性结构的方法对全机结构固有振动特性 进行计算, 表 2 给出了所关心频率范围内的主要模态 频率比, 这些数值是将真实频率除以参考频率 $f$ *得 到. 从计算结果中可以看出一些大展弦比复合材料 飞机的结构特点, 比如模态频率较为密集, 机身弯曲 频率较低等. 值得关注的一点是机翼对称扭转模态 为第 25 阶, 其频率比约为 19.64 , 可见该机翼经过了 细致的线性气动弹性剪裁设计. 机翼面内弯曲模态 频率较低, 但在线性气动弹性理论基础上该模态通 常不会参与气动弹性耦合.

采用频域偶极子格网法计算非定常气动力, 确 定颤振临界速度, 表 3 给出了线性颤振结果, 其中颤 振速度比为真实颤振速度与参考速度 $V *$ 的比值, 颤 振频率也用频率比来表示, 颤振 $V-f$ 和 $V-g$ 曲线如图 4 和 5 所示. 所得颤振速度为 112.7 , 远大于飞机平飞 的设计速度比 $V_{\mathrm{D}} / V^{*}$, 其值约为 68.5. 可见飞机在飞 行速度范围内不会发生颤振. 值得注意的是, 图 4 中 显示出第六阶机翼对称水平一弯模态已经略微的越 过了零线, 但变正后最大阻尼仅为约 $0.4 \%$ 且有下降 变负的趋势, 由于在计算中没有考虑结构阻尼以及 气动粘性阻尼, 该支模态通常认为不构成危险的颤 振分支. 这是由于机翼铺层设计使得结构具有一定 的水平弯曲和扭转方向的刚度耦合, 水平弯曲模态 振型具有一定的扭转运动分量, 因此造成了轻微的 气动弹性不稳定现象.

\section{表 1 线性情况平飞载荷翼尖垂向位移}

\begin{tabular}{cc}
\hline Displacement $(\mathrm{m})$ & Relative Disp. (\%) \\
\hline 0.5206 & 5.54 \\
\hline
\end{tabular}

\section{表 2 线性情况全机主要模态频率比}

\begin{tabular}{clc}
\hline Mode No. & \multicolumn{1}{c}{ Mode description } & $f^{*} f^{*}$ \\
\hline 1 & First symmetrical vertical bending of the wing & 1.414 \\
2 & First antisymmetric vertical bending of the wing & 2.368 \\
5 & Second symmetrical vertical bending of the wing & 4.718 \\
6 & First symmetrical chordwise bending of the wing & 5.379 \\
7 & First antisymmetric chordwise bending of the wing & 5.470 \\
8 & First vertical bending of the fuselage & 5.894 \\
9 & Second antisymmetric vertical bending of the wing & 6.320 \\
15 & Third symmetrical vertical bending of the wing & 10.798 \\
20 & Symmetrical outer aileron deflection & 14.476 \\
22 & Symmetrical inner aileron deflection & 15.286 \\
25 & First symmetrical torsion of the wing & 16.817 \\
\hline
\end{tabular}

表 3 线性颤振结果

\begin{tabular}{cc}
\hline$V_{\mathrm{F}} / V^{*}$ & $f_{\mathrm{F}} / f^{*}$ \\
\hline 112.7 & 7.78
\end{tabular}

Unstable mode: Second antisymmetric vertical bending of the wing

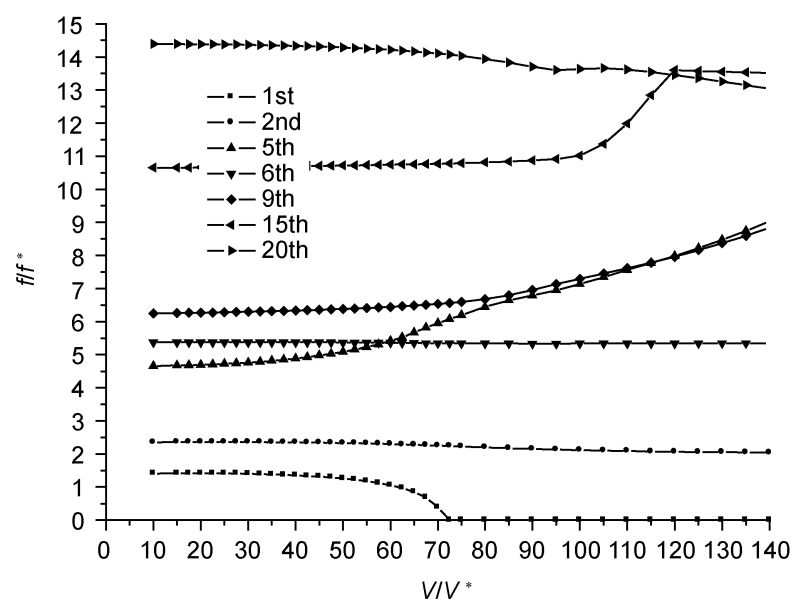

图 4 线性情况颤振 $V-f$ 曲线

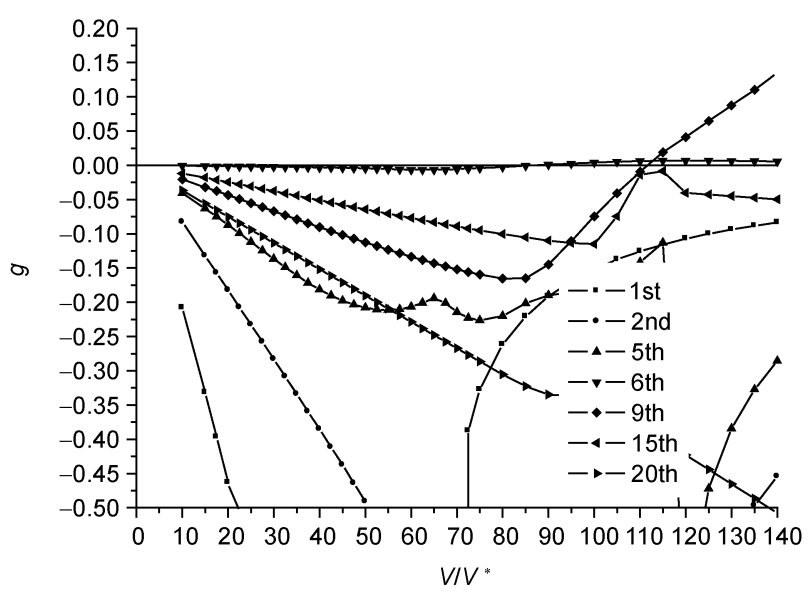

图 5 线性情况颤振 $V-g$ 曲线 


\section{2 平飞情况}

考虑结构几何非线性时, 首先对飞机在设计载 荷下平飞的气动弹性稳定性进行评估, 由于在考虑 几何非线性时结构变形和应力状态影响飞机的动力 学和气动弹性特性, 因此需要进行全机静-动力学耦 合求解, 从而判断全机非线性静平衡态的气动弹性 稳定性. 为简化计算在非线性分析时假定气动载荷大 小不变, 其方向则随气动面的弯曲而改变. 由于考虑 了载荷的随动效应, 在线性配平载荷作用下飞机并不 能保持平飞, 这里仅以刚体运动加速度作为配平量进 行静力学迭代计算. 表 4 给出了全机非线性静力学平 衡态的翼尖变形量, 与表 1 给出的线性计算相比略小, 这主要是由于相对线性计算而言全机有附加的惯性卸 载, 而且气动面弯曲后展向弯矩也会略微变小.

表 5 给出了平飞情况下全机非线性平衡态的前 若干阶弹性模态, 其中的频率比为真实频率与参考 频率 $f^{*}$ 的比值, 并且给出了这些模态频率与线性计 算所得结果的相对偏差. 除机翼面内弯曲频率有所 降低外, 其他频率均略有上升. 比较机翼面内弯曲和 扭转模态的频率偏差可以清楚的看出这两阶模态在 考虑几何非线性时的耦合关系, 即机翼面内弯曲频 率降低而扭转频率提高. 图 5 为机翼对称面内一弯模

\section{表 4 非线性情况平飞载荷翼尖垂向位移}

\begin{tabular}{cc}
\hline Displacement $(\mathrm{m})$ & Relative Disp. (\%) \\
\hline 0.5193 & 5.53 \\
Trim vertical acceleration $a_{y}\left(\mathrm{~m} / \mathrm{s}^{2}\right)$ & -0.12 \\
\hline
\end{tabular}

\section{表 5 平飞载荷情况主要模态频率比}

\begin{tabular}{|c|c|c|c|}
\hline Mode No. & Mode description & $f / f^{*}$ & Diff. (\%) \\
\hline 1 & $\begin{array}{l}\text { First symmetrical vertical } \\
\text { bending of the wing }\end{array}$ & 1.416 & 0.141 \\
\hline 2 & $\begin{array}{l}\text { First antisymmetric vertical } \\
\text { bending of the wing }\end{array}$ & 2.382 & 0.591 \\
\hline 5 & $\begin{array}{l}\text { Second symmetrical vertical } \\
\text { bending of the wing }\end{array}$ & 4.733 & 0.318 \\
\hline 6 & $\begin{array}{l}\text { First symmetrical chordwise } \\
\text { bending of the wing }\end{array}$ & 5.315 & -1.190 \\
\hline 7 & $\begin{array}{l}\text { First antisymmetric chordwise } \\
\text { bending of the wing }\end{array}$ & 5.425 & -0.823 \\
\hline 8 & First vertical bending of the fuselage & 5.938 & 0.747 \\
\hline 9 & $\begin{array}{l}\text { Second antisymmetric vertical } \\
\text { bending of the wing }\end{array}$ & 6.338 & 0.285 \\
\hline 15 & $\begin{array}{l}\text { Third symmetrical vertical } \\
\text { bending of the wing }\end{array}$ & 10.819 & 0.194 \\
\hline 20 & Symmetrical outer aileron deflection & 14.519 & 0.297 \\
\hline 22 & Symmetrical inner aileron deflection & 15.292 & 0.039 \\
\hline 25 & First symmetrical torsion of the wing & 17.005 & 1.118 \\
\hline
\end{tabular}

态的振型图, 几何非线性的作用还使得该模态振型 具有了相当明显的机翼扭转运动分量.

利用全机非线性静变形后的模态，通过偶极子 格网法计算频域非定常气动力, 判断全机气动弹性 稳定性. 表 6 给出了考虑结构大变形几何非线性的颤 振计算结果, 其中颤振速度比为颤振速度计算值与 参考速度 $V^{*}$ 的比值, 颤振频率比为频率计算值与参 考频率 $f^{*}$ 的比值. 计算采用的定常气动载荷对应的 飞行速度 $V_{\mathrm{D}}$ 与 $V^{*}$ 比值约为 68.5. 从表中可以看出, 预测颤振临界速度比为 68.7, 略微大于飞行速度. 由 于系统真实的颤振临界速度位于其预测值与定常载 荷对应的飞行速度值之间, 因此对本算例而言系统 确切的颤振临界速度比可以认为就是 68.7, 因此系 统是气动弹性稳定的. 颤振的失稳模态为机翼对称 面内一弯, 参与耦合的主要模态为机翼对称垂直一 弯与机翼对称垂直二弯, 在线化模态计算中已经提 到机翼结构在承载后水平弯曲模态的振型具有了显 著的扭转运动分量, 因此它与临近的垂直弯曲模态 相互作用出现了颤振现象, 由于各阶耦合模态频率 较低造成了远远低于线性情况的颤振临界速度, 而 这一颤振分支在线性分析时是不存在的. 其次, 全机 的第二个颤振分支为机翼反对称垂直二弯, 其耦合 类型与线性情况相同, 主要的参与模态为副翼偏转 模态和机翼扭转模态, 从其失稳速度可以看出它与 线性情况差异不大. 该阶鲴振类型主要以高阶模态 为主，而大变形几何非线性因素对高阶模态的影响 非常小, 因此并没有引起其失稳速度的大幅改变. 由

\section{表 6 平飞情况非线性颤振计算结果}

\begin{tabular}{ccc}
\hline Flutter No. & $V_{\mathrm{F}} / V^{*}$ & $f_{\mathrm{F}} / f^{*}$ \\
\hline \multirow{2}{*}{1} & 68.7 & 5.23 \\
& First symmetrical chordwise bending of the wing \\
2 & 114.4 & 7.83 \\
& Second antisymmetric vertical bending of the wing \\
\hline
\end{tabular}

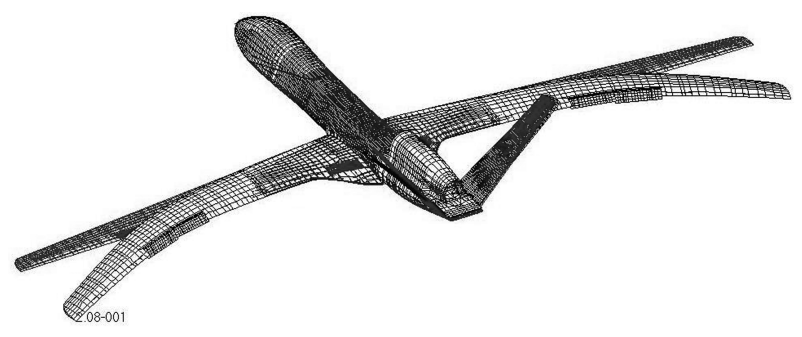

图 6 非线性情况机翼对称面内一弯振型图 
于飞行载荷和变形都是对称的, 因此对称模态与非 对称模态之间不存在气动弹性耦合.

图 7 和 8 为计算得到的全机主要模态 $V-f$ 和 $V-g$ 曲线, 图中用坚直虚线标出了定常气动力对应的飞 行速度比 $V_{\mathrm{D}} / V^{*}$, 穿越零阻尼线的模态有两支, 具有 较低速度的是机翼对称水平一弯模态. 从全图可以 看出全机气动弹性稳定性的总体趋势, 除机翼对称水 平一弯模态外, 其他各阶模态曲线与线性分析中的 $V-g$ 曲线类似. 机翼对称水平一弯模态对应的曲线也 具有其显著特点, 当来流速度很小时该模态以面内 的水平运动为主, 该模态坐标上基本没有广义气动 力分量, 即结构在该模态上几乎不存在与气流的能 量交换, 因此在 $V-g$ 曲线上表现为接近零阻尼的直线; 当来流速度进一步提高即结构变形增大, 水平模态

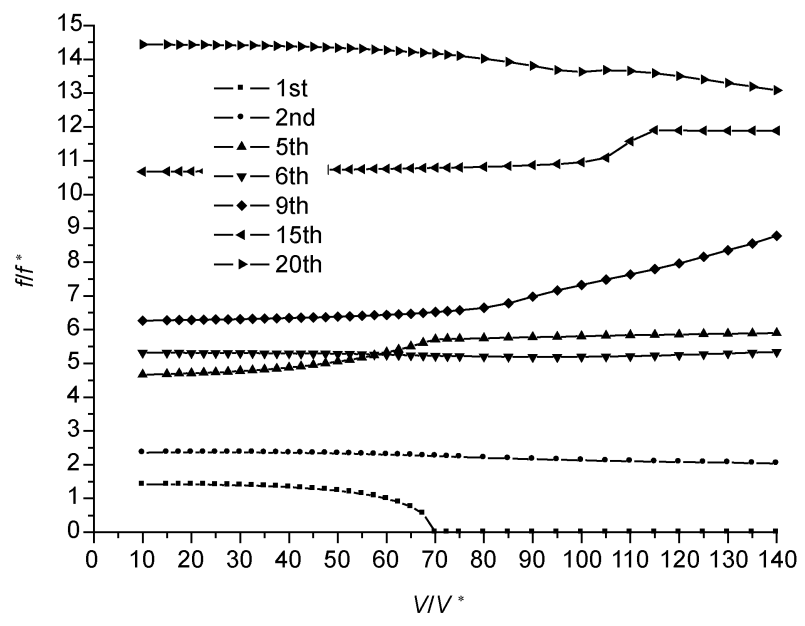

图 7 平飞情况非线性颤振 $V-f$ 曲线

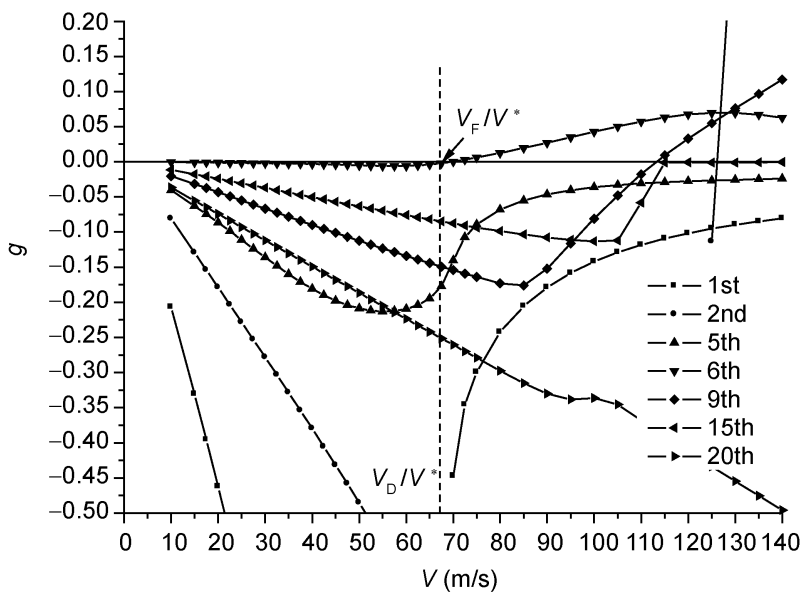

图 8 平飞情况非线性颤振 $V-g$ 曲线
的扭转运动分量逐渐显著, 广义气动力在该模态上 的分量也增大起来, 能量交换也不断加强, 进一步与 相关的垂直弯曲模态形成气动弹性的不利耦合就出 现了气动弹性不稳定, 即产生了颤振.

这里需要说明的一个重要问题是, 以上的分析 过程是在指定的载荷情况即飞行速度为 $V_{\mathrm{D}}$ 时的全机 平衡态进行线性化的, 因此图 6 中的确切可信部分仅 为 $V_{\mathrm{D}} / V^{*}$ 虚线的左右邻域范围, 其他区域仅说明各阶 模态阻尼随飞行速度变化的大致趋势. 尤其是当计 算得到的最低颤振临界速度没有落在该邻域内时, 所得值仅为预测值而并不是系统确切的颤振临界速 度, 确切值应位于飞行速度与颤振速度预测值之间. 为得到系统的确切颤振临界速度或完整的 $V-g$ 曲线 就需要进行迭代计算或将飞行速度进行一定的划分 来进行搜索计算. 本算例的颤振速度预测值恰好落 在 $V_{\mathrm{D}} / V^{*}$ 虚线附近, 因此可以认为已经得到了系统确 切的颤振临界速度.

\section{3 突风情况}

前节中对全机平飞情况进行了几何非线性气动 弹性稳定性分析, 颤振速度预测值恰好约等于平飞速 度, 当然按照飞机设计规范并不能满足稳定余度要求. 然而, 在考虑结构几何非线性时全机颤振特性受载荷 状态和变形量的影响, 这就需要讨论在遇到突风这种 突发的严重载荷情况下飞机的气动弹性稳定性.

本文采用等效的静态载荷计算全机阵风情况的 变形, 表 7 给出了计算结果, 此时翼尖垂直位移量达 到了半翼展的 $10 \%$, 可见大变形的几何非线性效应必 然是不能忽略的重要因素. 表 8 给出了突风载荷下全 机的主要弹性模态频率计算结果, 以及相对线性计算 的偏差. 与表 5 结果对比可以看到, 由于载荷和变形 量均大于平飞情况, 几何非线性对各阶模态的影响也 更为显著, 面内弯曲频率下降更为明显, 并且机翼面 内弯曲模态的振型也具有更为显著的扭转运动分量.

表 9 给出了突风情况下的颤振计算结果, 相应的 $V-f$ 和 $V-g$ 曲线如图 9 和 10 所示. 其危险颤振分支为机 翼对称面内一弯, 颤振临界速度比的预测值仅为 55.3 , 小

\section{表 7 纵向突风翼尖垂向位移}

\begin{tabular}{ccc}
\hline Calculation Case & Linear & Nonlinear \\
\hline Displacement (m) & 1.014 & 1.004 \\
Relative Disp. (\%) & 10.81 & 10.70 \\
\hline
\end{tabular}


表 8 突风载荷情况主要模态频率比

\begin{tabular}{|c|c|c|c|}
\hline Mode No. & Mode description & $f / f^{*}$ & Diff. $(\%)$ \\
\hline 1 & First symmetrical vertical bending of the wing & 1.416 & 0.141 \\
\hline 2 & First antisymmetric vertical bending of the wing & 2.394 & 1.098 \\
\hline 5 & Second symmetrical vertical bending of the wing & 4.722 & 0.074 \\
\hline 6 & First symmetrical chordwise bending of the wing & 5.170 & -3.885 \\
\hline 7 & First antisymmetric chordwise bending of the wing & 5.320 & -2.751 \\
\hline 8 & First vertical bending of the fuselage & 5.921 & 0.450 \\
\hline 9 & Second antisymmetric vertical bending of the wing & 6.341 & 0.324 \\
\hline 15 & Third symmetrical vertical bending of the wing & 10.779 & -0.176 \\
\hline 20 & Symmetrical outer aileron deflection & 14.543 & 0.463 \\
\hline 22 & Symmetrical inner aileron deflection & 15.303 & 0.108 \\
\hline 25 & First symmetrical torsion of the wing & 17.163 & 2.054 \\
\hline
\end{tabular}

\section{表 9 突风情况非线性颤振计算结果}

\begin{tabular}{ccc}
\hline Flutter No. & $V_{\mathrm{F}} / V^{*}$ & $f_{\mathrm{F}} / f^{*}$ \\
\hline \multirow{2}{*}{1} & 55.3 & 5.08 \\
& First symmetrical chordwise bending of the wing \\
2 & 117.0 & 7.93 \\
& Second antisymmetric verticalbending of the wing \\
\hline
\end{tabular}

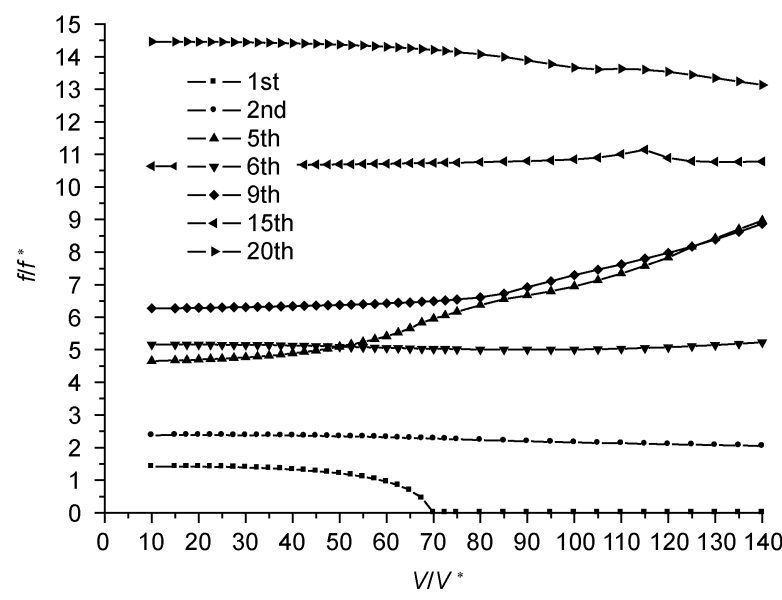

图 9 突风情况非线性㗜振 $V-f$ 曲线

于飞行速度比 68.5 , 真实的颤振速度位于两者之间, 因此全机在平飞遭遇突风情况下是气动弹性不稳定 的. 第二个颤振分支为机翼反对称垂直二弯模态, 其 颤振临界速度与线性计算结果接近.

对比平飞情况和突风情况的计算结果可以看到, 在考虑结构非线性因素时, 飞机的受载状态对其颤振 特性具有一定影响, 因此飞机的颤振特性是与飞行状 态相关的特性，这与传统线性观点的认识有所不同.

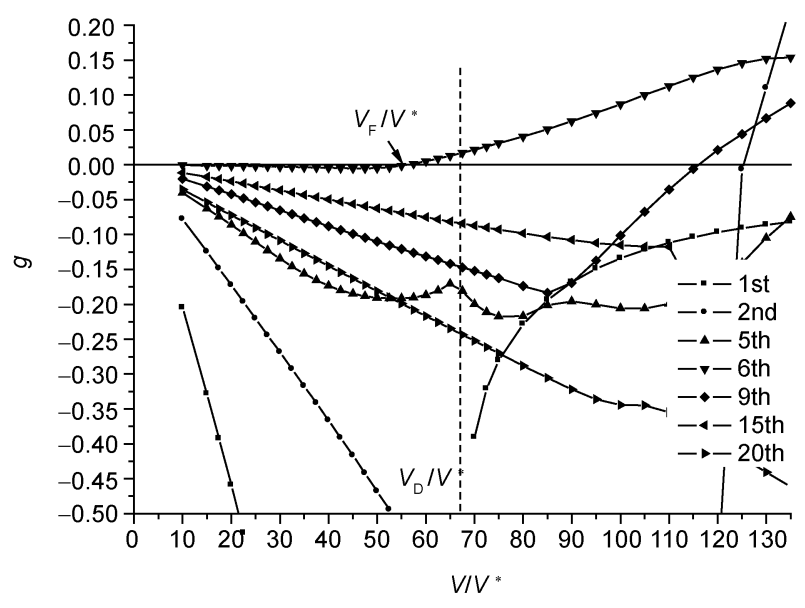

图 10 突风情况非线性颤振 $V-g$ 曲线

\section{5 结论}

本文针对具有大展弦比复合材料机翼的某无人 机进行了自由飞行的非线性配平计算, 并通过小振幅 线性化方法考虑了结构变形和内应力对系统刚度的影 响, 形成了全机几何非线性气动弹性稳定性分析的工 程实施方法. 计算表明结构几何非线性对具有大展弦 比机翼的飞机气动弹性特性有可能造成严重影响, 使 得颤振速度降低, 甚至会产生线性分析中不会出现的 颤振分支. 对所研究的对象, 结构承载后由于几何非 线性的作用使得机翼水平一弯模态振型具有了显著的 扭转运动分量, 从而形成了机翼水平一弯与机翼垂直 一弯及机翼垂直二弯相互耦合的颤振，并且颤振速度 远低于线性分析结果. 当然, 本文的稳定性分析中忽 略了模态阻尼, 特别是对复合材料机翼面内弯曲这样 
的运动其结构阻尼和气动阻尼有可能对改善稳定性起 到重要的作用. 但是结构阻尼和气动阻尼通常难以准 确获得, 忽略这些因素将给出较为保守的稳定性预测.

应用本文所建立的几何非线性气动弹性稳定性 分析的线性化方法和工程求解流程, 验证了线性化理 论和方法对于解决复杂工程问题的可用性和实用性,
并研究了典型大柔性飞行器的几何非线性气动弹性特 性. 从研究结果来看, 对于具有大展弦比机翼的高空 长航时无人机的设计, 传统的线性分析方法不但不能 得到准确的颤振速度甚至有可能造成严重错误, 因此 几何非线性气动弹性稳定性分析有必要成为工程实施 的必须任务.

\section{参考文献}

1 van Schoor M C, von Flotow A H. Aeroelastic characteristics of a highly flexible aircraft. J Aircraft, 1990, 27: 901-908

2 Patil M J. Nonlinear Aeroelastic Analysis, Flight Dynamics, and Control of a Complete Aircraft. Dissertation of Doctoral Degree. Atlanta: Georgia Institute of Technology, 1999

3 Mayuresh J P, Dewey H H. Nonlinear aeroelasticity and flight dynamics of high-altitude long-endurance aircraft. J Aircraft, 2001, 38: 88-94

4 Dowell E, Edwards J, Strganac T. Nonlinear aeroelasticity. J Aircraft, 2003, 40: 857-874

5 Mayuresh J P, Dewey H H. On the importance of aerodynamic and structural nonlinearities in aeroelastic behavior of high-aspect-ratio wings. 41st AIAA/ASME/ASCE/AHS/ASC Structures, Structural Dynamics, and Materials Conference and Exhibit, Atlanta, 2000. AIAA-2000-1448

6 Tang D, Dowell E H. Experimental and theoretical study on aeroelastic response of high-aspect-ratio wings. AIAA J, 2001, 39: $1430-1441$

7 谢长川. 飞行器气动弹性稳定性静/动耦合理论与试验研究. 博士学位论文. 北京: 北京航空航天大学, 2009

8 张健. 柔性飞机非线性气动弹性与飞行动力学耦合建模与仿真. 博士学位论文. 北京: 北京航空航天大学, 2010

9 Cesnik C E S, Su W H. Nonlinear aeroelastic modeling and analysis of fully flexible aircraft. 46th AIAA/ASME/ASCE/AHS/ASC Structures, Structural Dynamics, and Materials Conference, Austin, 2005, AIAA2005-2169

10 Chang C S, Dewey H H, Mayuresh J P. Flight dynamics of highly flexible aircraft. J Aircraft, 2008, 45: 538-545

11 Shearer C M, Cesnik C E S. Nonllinear flight dynamics of very flexible aircraft. J Aircraft, 2007, 44: 1528-1545

12 Strong D D, Kolonay R M, Huttsell L J, et al. Flutter analysis of wing configurations using pre-stressed frequencies and mode shapes. 46th AIAA/ASME/ASCE/AHS/ASC Structures, Structural Dynamics, and Materials Conference, Austin, 2005, AIAA 2005-2173

13 谢长川, 吴志刚, 杨超. 大展弦比柔性机翼的气动弹性分析. 北京航空航天大学学报, 2003, 29: 1087-1090

14 Xie C C, Leng J Z, Yang C. Geometrical nonlinear aeroelastic stability analysis of a composite high-aspect-ratio wing. Shock Vib, 2008, 15: $325-333$

15 冷佳桢, 谢长川, 杨超. 水平弯曲刚度对大展弦比机翼颤振的影响. 北京航空航天大学学报, 2009, 35: 718-722

16 Rodden W P, Johnson E H. MSC/NASTRAN Version 68 Aeroelastic Analysis Users Guide. New York: The MacNeal-Schwendler Corporation, 1994

17 王昮成, 邵敏. 有限单元法基本原理和数值方法. 北京: 清华大学出版社, 1997

18 管德. 非定常空气动力计算. 北京: 北京航空航天大学出版社, 1991

19 Shirk M H, Hertz T J, Weisshaar T A. Aeroelastic tailoring - theory, practice, and promise. J Aircraft, 1986, 23: 6-18 